\title{
Memory Retrieval and the Passage of Time: From Reconsolidation and Strengthening to Extinction
}

\author{
Maria Carmen Inda, ${ }^{1}$ Elizaveta V. Muravieva, ${ }^{1}$ and Cristina M. Alberini ${ }^{1,2}$ \\ Departments of ${ }^{1}$ Neuroscience and ${ }^{2}$ Psychiatry, Mount Sinai School of Medicine, New York, New York, 10029
}

An established memory can be made transiently labile if retrieved or reactivated. Over time, it becomes again resistant to disruption and this process that renders the memory stable is termed reconsolidation. The reasons why a memory becomes labile after retrieval and reconsolidates still remains debated. Here, using inhibitory avoidance learning in rats, we provide evidence that retrievals of a young memory, which are accompanied by its reconsolidation, result in memory strengthening and contribute to its overall consolidation. This function associated to reconsolidation is temporally limited. With the passage of time, the stored memory undergoes important changes, as revealed by the behavioral outcomes of its retrieval. Over time, without explicit retrievals, memory first strengthens and becomes refractory to both retrieval-dependent interference and strengthening. At later times, the same retrievals that lead to reconsolidation of a young memory extinguish an older memory. We conclude that the storage of information is very dynamic and that its temporal evolution regulates behavioral outcomes. These results are important for potential clinical applications.

\section{Introduction}

A newly formed memory is initially labile and can be disrupted by a variety of interferences, including inhibition of new protein synthesis, gene expression, and inactivation of brain regions (Davis and Squire, 1984; Dudai and Eisenberg, 2004). Over time, memory becomes resistant to disruption through a process known as consolidation (McGaugh, 2000). Different types of interferences suggest the existence of multiple phases, or processes, underlying the temporal evolution of consolidation. For example, for hippocampal-dependent memories, hippocampal inhibition of protein/RNA synthesis indicates that the gene expressiondependent phase of consolidation is completed within the first 1-2 d (Medina et al., 2008; Alberini, 2009). In contrast, hippocampal lesions or inactivation result in amnesia for experiences that occurred several weeks (in animals) or years (in humans) before, whereas similar memories of older events remain intact; thus, it has been proposed that memory consolidation reflects a processing of network rearrangement throughout the brain (Frankland and Bontempi, 2005; Moscovitch et al., 2006; Squire and Bayley, 2007).

In the last 10 years, it became clear that the protein- and RNA synthesis-dependent phase of memory consolidation is not restricted to the first day after training, but can occur again, even several days or weeks following training, if the memory is retrieved or reactivated (Nader et al., 2000; Sara, 2000a). The reactivated, labile memory stabilizes again through a process termed

Received Sept. 9, 2010; revised Nov. 22, 2010; accepted Dec. 2, 2010.

This work was supported by National Institute of Mental Health-National Institutes of Health Grant R01MH074736 awarded to C.M.A., and Human Frontiers Science Program long-term fellowship LT-00861/2008-L to M.C.I. We thank Gabriella Pollonini, Reginald Miller, and the Center for Comparative Medicine and Surgery Facility at Mount Sinai School of Medicine for technical support.

Correspondence should be addressed to Cristina M. Alberini, Department of Neuroscience, Box 1065, Mount Sinai School of Medicine, New York, NY 10029. E-mail: cristina.alberini@mssm.edu.

DOI:10.1523/JNEUROSCI.4736-10.2011

Copyright $\odot 2011$ the authors $\quad 0270-6474 / 11 / 311635-09 \$ 15.00 / 0$ reconsolidation (Nader et al., 2000; Sara, 2000a). This postretrieval lability has been found with numerous memory tasks and in different species, although in some conditions it was not observed (Dawson and McGaugh, 1969; Tronson and Taylor, 2007; for review, see Alberini, 2005). In several cases (Milekic and Alberini, 2002; Eisenberg and Dudai, 2004; Suzuki et al., 2004; Frankland et al., 2006; Bustos et al., 2009), but not all (Debiec et al., 2002), memory shows an age-dependent, temporally graded resistance to post-retrieval disruption, suggesting that reconsolidation may reflect a phase of a lingering consolidation process (Dudai and Eisenberg, 2004; Alberini, 2005). Importantly, retrieval or reactivation of a reinforced associative memory generally consists of an exposure to the conditioned stimulus (CS) alone, without the reexperiencing of the reinforcing unconditioned stimulus (US), a procedure known to also induce extinction, that is a decrease in the conditioned response (Quirk and Mueller, 2008).

Why does memory undergo retrieval-dependent reconsolidation and why is reconsolidation temporally graded? Two hypotheses had been proposed. One posits that memory reconsolidates to update or add new information to an established memory (Lewis, 1979; Sara, 2000a; Dudai, 2004), which implies that reconsolidation can occur at any time during the life of a memory; the second proposes that memory reconsolidates to strengthen (Sara, 2000b).

Although in some experimental conditions reconsolidation occurs in parallel with memory updating (Rodriguez-Ortiz et al., 2005, 2008; Morris et al., 2006; Hupbach et al., 2007), a formal demonstration that reconsolidation mediates memory updating is still debated (Lee, 2009; Suarez et al., 2010). Furthermore, although a recent study showed that undergoing a reinforced experience (retraining) makes the memory labile and leads to memory strengthening (Lee, 2008), the function of retrievalinduced reconsolidation remains to be established. It also re- 
mains to be understood why the post-retrieval fragility changes with time.

In this study, using rat inhibitory avoidance (IA), we tested whether the post-retrieval memory fragility, or reconsolidation, mediates memory strengthening and its interaction with the passage of time.

\section{Materials and Methods}

Animals. Adult male Long-Evans rats weighing between 200 and $250 \mathrm{~g}$ were used in all experiments. Animals were housed and maintained on a $12 \mathrm{~h}$ on/12 h off light/dark cycle. All rats were allowed free access to food and water. The animals were handled for $5 \mathrm{~d}$ before behavioral procedures. All protocols complied with the National Institutes of Health Guide for the Care and Use of Laboratory Animals and were approved by the Mt. Sinai School of Medicine Animal Care Committees.

Inhibitory avoidance. The IA chamber consisted of a rectangularshaped box with two compartments, a safe (lit) and a shock (dark) one, separated by a sliding door (model ENV-010MC; Med Associates). The chamber was located in a sound-attenuated, nonilluminated room. The experiments were conducted as previously described (Milekic and Alberini, 2002). During training, each rat was placed in the safe compartment facing away from the door. After $10 \mathrm{~s}$, the door opened, allowing the rat access to the shock compartment, where a brief footshock $(0.6 \mathrm{~mA}$, $2 \mathrm{~s}$ ) was delivered. Latency to enter the shock compartment was taken as a measure of acquisition. The rat was then returned to its home cage and memory retention was tested at the indicated times as described in each experiment. During the retention test, the animal was placed in the safe compartment and $10 \mathrm{~s}$ later, the door opened. Latency to enter into the shock compartment was measured in seconds. No footshock was delivered during testing, which was terminated at $900 \mathrm{~s}$. Training and testing procedures were performed blind to treatments. Memory reactivation consisted of either testing or $10 \mathrm{~s}$ exposures to the safe compartment, as specified in Results. In a control experiment $10 \mathrm{~s}$ exposures to a different context (context B) were used. Context B consisted of a square chamber $(43.2 \mathrm{~cm} \times 43.2 \mathrm{~cm} \times 43.2 \mathrm{~cm}$, model ENV-515 Med Associates) with one transparent and three opaque Plexiglas walls and a Plexiglas flat floor. To test whether memory impairment was due to extinction or reconsolidation, animals underwent a reminder footshock $(0.6 \mathrm{~mA})$ in a different context.

Drug administration. Systemic injections of cycloheximide (SigmaAldrich Inc) were performed in a manner similar to those described in other studies (Milekic et al., 2006). Rats were subcutaneously injected with either $2.2 \mathrm{mg}$ of cycloheximide/ $\mathrm{kg}$ of body weight or an equivalent volume of vehicle solution. Cycloheximide was dissolved in DMSO and finally diluted to $1 \%$ DMSO in saline. Vehicle solutions were prepared accordingly. This dose has been previously shown to disrupt memory consolidation and reconsolidation (Davis and Squire, 1984; Milekic and Alberini, 2002).

Statistical analyses. Statistical analyses were performed using either two-way ANOVA followed by Bonferroni post hoc test, one-way ANOVA followed by Newman-Keuls post hoc test, or, for pairwise comparisons, a Student's $t$ test.

\section{Results}

\section{Retrieval strengthens the retention of a young memory}

Previous studies have shown that IA memory of trained rats exposed to the context without shock during testing, $2 \mathrm{~d}$ after retrieval, becomes sensitive to disruption by several amnestic agents including protein synthesis inhibitors (Taubenfeld et al., 2001; Milekic and Alberini, 2002; Boccia et al., 2007; Taubenfeld et al., 2009). During IA testing, the animal is placed into the lit (safe) chamber, and the latency to enter into the dark (previously paired with a footshock) chamber is recorded. Testing is terminated after the animal enters the shock compartment or at a predetermined cutoff time. Hence, at testing, some animals enter the shock chamber before the cutoff time, whereas others do not. In IA, as in other fear conditioning tasks, retrieval by repeated CS exposures evokes extinction learning, which is a decrease in the conditioned response (Quirk and Mueller, 2008). Notably, a short or long duration of the CS exposure has been shown to preferentially lead to either reconsolidation or extinction, respectively (Pedreira and Maldonado, 2003; Suzuki et al., 2004; Power et al., 2006). Thus, in IA, the time spent in the lit chamber as well as the reexperience of the dark chamber during a nonreinforced retrieval may preferentially drive the memory toward reconsolidation or extinction, which is in agreement with the hypothesis of trace dominance (Eisenberg et al., 2003).

In the first sets of experiments, we tested the effect of a single reactivation consisting of either full testing (T) or a brief, $10 \mathrm{~s}$ exposure to the lit chamber ( $10 \mathrm{~s}$ ) on memory retention. Rats that were trained at the same time and remained in the home cage served as no-reactivation (NR) controls. Rats were trained, underwent reactivation $2 \mathrm{~d}$ later, and were tested after $2 \mathrm{~d}$. As shown in Figure 1, $A$ and $B$, the retention of rats that underwent either type of reactivation was similar to those of the respective NR groups. The retention values \pm SEM and number of rats per group of all experiments presented in this paper are shown in Table 1. One-way ANOVA across groups $\left(F_{(2,27)}=0.6549, p=\right.$ 0.5275 , Fig. $1 A)$ or Student's $t$ test $\left(t_{(12)}=0.2266, p=0.8246\right.$, Fig. $1 B)$ revealed no significant effect of reactivation. In experiments using one $\mathrm{T}$, we observed that the final retention latency varied in each animal, showing sometimes a tendency toward a decrease and at other times a tendency toward an increase in retention when compared with their latency of the reactivating test (T1) (Fig. 1A), although these differences were never significant. We hypothesized that, because during a full test the animal remains in the apparatus for several minutes and may eventually enter the shock chamber, some animals may extinguish and others may reconsolidate the memory, according to the hypothesis of trace dominance (Dudai, 2006). Hence, to amplify the outcome, we investigated the effect of multiple reactivations.

Rats were trained and underwent either three reactivations by testing $(3 \times \mathrm{T})$ or $10 \mathrm{~s}(3 \times 10 \mathrm{~s})$ with an interreactivation interval of $2 \mathrm{~d}$ and, $2 \mathrm{~d}$ later, were finally tested ( $8 \mathrm{~d}$ after training, Fig. $1 C, D)$. As shown in Figure $1 C, 3 \times \mathrm{T}$ led to memory decrease. One-way ANOVA that compared the latencies across groups revealed a significant effect of reactivations $\left(F_{(4,24)}=6.634, p=\right.$ $0.0010)$. Newman-Keuls post hoc test showed that, at final testing, the $3 \times \mathrm{T}$ group had a significantly lower memory retention compared with NR $(p<0.01)$. To investigate whether this decreased memory was due to extinction, $24 \mathrm{~h}$ after the last test, the rats underwent a reminder footshock in a different context, to explore whether memory retention could be reinstated (Bouton, 1993). As shown in Figure $1 C, 24 \mathrm{~h}$ after the reminder, memory retention was significantly reinstated to NR control levels (Student's $t$ test, $\left.t_{(10)}=2.443, p=0.0347\right)$ indicating that the decreased retention resulted from $3 \times \mathrm{T}$ reactivations was due to extinction. In contrast, trained rats that underwent $3 \times 10 \mathrm{~s}$ reactivations had a significantly increased memory retention compared with NR controls and with $3 \times 10 \mathrm{~s}$ exposures into a different context (context B, Fig. 1D). A one-way ANOVA comparing latencies at testing revealed a significant effect of reactivations $\left(F_{(2,25)}=11.31, p=0.0003\right)$. A Newman-Keuls' post hoc test revealed that $3 \times 10 \mathrm{~s}$ reactivations lead to a significant increase in latency compared with either NR $(p<0.001)$ or $3 \times 10$ s exposures to a different context $(p<0.01)$. Hence, whereas three reactivations by full testing lead to extinction, three reactivations by brief, $10 \mathrm{~s}$ exposure to the lit compartment lead to memory strengthening.

We then asked whether spacing the interreactivation interval between testing could lead to memory strengthening rather than 
A
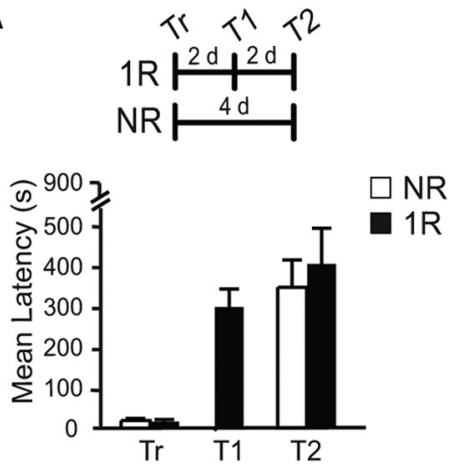

C
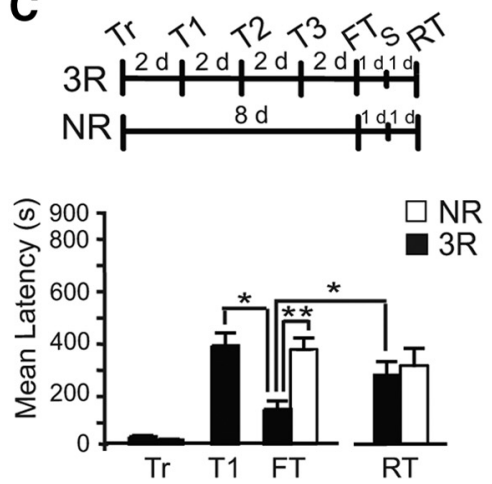

$\mathbf{E}$

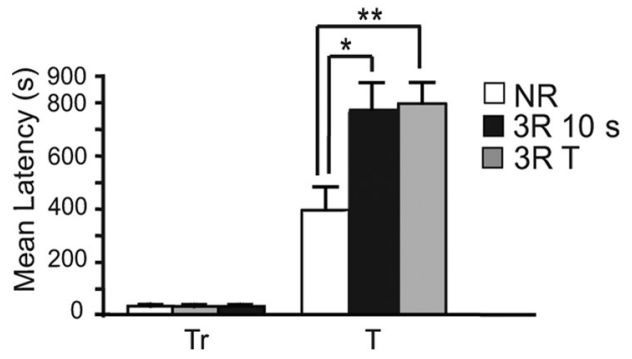

Figure 1. Multiple reactivations result in memory strengthening. $\boldsymbol{A}, \boldsymbol{B}$, Experimental timelines for each panel are shown. Memory acquisition (Tr) and retention are expressed as mean latency \pm SEM (in seconds, $s$ ). Rats were trained (Tr) and either reactivated $(1 \mathrm{R})$ by testing $(\mathrm{T} 1, \boldsymbol{A})$ or 10 s context exposure $(\boldsymbol{B}) 2 \mathrm{~d}$ after training, or remained in the home cage (NR). All rats were tested $4 \mathrm{~d}$ after training. At testing, no significant differences in latencies were found between each $1 \mathrm{R}$ and NR groups. $\boldsymbol{C}$, Rats were trained and underwent reactivation by testing 3 times with an interreactivation interval of $2 \mathrm{~d}(3 \mathrm{R})$. A control group was trained and remained in the home cage (NR). All animals were tested $8 \mathrm{~d}$ after training (final test, FT). One day later, they received a reminder footshock (S) and, were re tested $24 \mathrm{~h}$ later (RT). FT of rats that underwent reactivations showed a significant latency decrease compared with $\mathrm{T} 1$ or NR $\left({ }^{* *} p<0.01\right)$ that was reinstated by $S\left({ }^{*} p<0.05\right)$. D, Rats were trained and, 2 d later, reactivated by three 10 s context exposures with 2 interreactivation interval $(3 \times 10 \mathrm{~s})$; a control group was trained and received three 10 s exposures to a different context (Context B, Cnt), another control group remained in the home cage (NR). At testing $(\boldsymbol{T})$, the $3 \times 10$ s group showed a significant increase in latency compared with the NR group $\left({ }^{* *} p<0.01\right)$. $\boldsymbol{E}$, Rats were trained and reactivated, by either testing $(3 \times \mathrm{T})$, or $3 \times 10 \mathrm{~s}$, with an interreactivation interval of 1week, or remained in the home cage (NR). Both groups that underwent either type of reactivations showed a significant increase in latency compared with NR $\left({ }^{*} p<0.05,{ }^{* *} p<0.01\right)$.

significantly enhanced memory retention compared with the NR $(p<0.01$ for $3 \times \mathrm{T}$ and $p<0.05$ for $3 \times 10 s$ ).

Hence, retrievals that favor reconsolidation and limit extinction, such as short exposures to the CS or retrievals spaced by long intervals of time, leads to memory strengthening. We used the $3 \times 10 \mathrm{~s}$ paradigm to investigate the functional outcomes of memory reactivation.

\section{A protein synthesis-dependent reconsolidation process mediates memory strengthening}

Previous studies showed that reconsolidation is not only dependent on the age of the memory, but also on the intensity of training and recall (Milekic and Alberini, 2002; Eisenberg et al., 2003; Suzuki et al., 2004; Bustos et al., 2009), and suggested that a very brief reactivation may not result in memory fragility and, therefore, reconsolidation (Suzuki et al., 2004; Bustos et al., 2009). Thus, we tested whether the $3 \times 10$ s reactivations, which result in significant memory strengthening, induce a protein synthesis-dependent reconsolidation process. Rats were trained and underwent either $3 \times 10$ s reactivations with an interreactivation interval of $2 \mathrm{~d}$ or remained in the home cage (NR). Each group received a systemic, subcutaneous injection of either cycloheximide or vehicle solution either after each reactivation or at the paired time points in the NR groups (Fig. 2B). We previously established that this subcutaneous cycloheximide injection blocks $>70 \%$ of new protein synthesis $1 \mathrm{~h}$ after injection and $23 \% 6 \mathrm{~h}$ after injection (Milekic et al., 2006). All rats were tested $2 \mathrm{~d}$ after the last reactivation ( $8 \mathrm{~d}$ after training). Although it should be acknowledge that protein synthesis inhibitors may exert some secondary effects, the amnesia resulting from protein synthesis inhibition during the posttraining or post-retrieval phases has been established using several different inhibitors, each with different secondary effects as well as several molecular targeted

extinction. Hence, we compared the effect of multiple retrievals by either $3 \times$ T or $3 \times 10$ s given with an interreactivation interval of 1 week.

Rats were trained and divided into three groups. One group received $3 \times 10 \mathrm{~s}$, the second received $3 \times \mathrm{T}$, and the third remained in the home cage (NR, Fig. $1 E$ ). Two days after the last reactivation, the animals were tested. NR rats were tested in parallel, $23 \mathrm{~d}$ after training. As depicted in Figure $1 E$, both groups that received three reactivations had a remarkable increase in memory retention compared with the NR group. A one-way ANOVA comparing the latencies across groups showed a significant effect of reactivations $\left(F_{(2,14)}=6.238, p=0.0116\right)$. Newman-Keuls post hoc test revealed that the reactivations approaches (Alberini, 2008). As shown in Figure 2A, a two-way ANOVA revealed a significant effect of treatment $\left(F_{(1,43)}=27.69\right.$, $p<0.0001)$ and treatment $\times$ reactivation interaction $\left(F_{(1,43)}=\right.$ $22.68, p<0.0001)$. Bonferroni post hoc test showed that in agreement with our previous data shown in Figure $1 D$, compared with the NR-vehicle-injected group, the $3 \times 10$ s reactivated-vehicleinjected group had significantly higher memory retention $(p<$ $0.01)$. This retention, however, was significantly disrupted by $c y-$ cloheximide $(p<0.001)$. Furthermore, the $3 \times 10$ s reactivated cycloheximide-injected group had a significantly lower retention compared with that of NR-cycloheximide-injected group $(p<$ 0.01 ), suggesting that cycloheximide disrupts memory reconsolidation evoked by the 3 reactivations. No effect of cyclohexi- 


\begin{tabular}{|c|c|c|c|c|c|c|c|}
\hline & \multirow[b]{2}{*}{$N$} & \multicolumn{6}{|c|}{ Latency (seconds) } \\
\hline & & $\operatorname{Tr}$ & $\mathrm{T}$ & $\mathrm{T} 1$ & $\mathrm{~T} 2$ & $\mathrm{FT}$ & RT \\
\hline \multicolumn{8}{|l|}{ Figure 1} \\
\hline \multirow[t]{2}{*}{$A$} & $\mathrm{NR} n=8$ & $18.7 \pm 5.8$ & & - & $351.0 \pm 68.2$ & & \\
\hline & $1 \mathrm{R} n=11$ & $18.0 \pm 3.5$ & & $303.5 \pm 42.9$ & $410.1 \pm 86.8$ & & \\
\hline \multirow[t]{2}{*}{ B } & $\mathrm{NR} n=7$ & $18.5 \pm 1.5$ & & $395.5 \pm 80.6$ & & & \\
\hline & $1 \mathrm{R} n=7$ & $22.3 \pm 2.0$ & & $371.5 \pm 68.7$ & & & \\
\hline \multirow[t]{2}{*}{$C$} & $\operatorname{NR} n=6$ & $12.6 \pm 2.1$ & & - & & $314.9 \pm 34.9$ & $244.3 \pm 52.9$ \\
\hline & $3 R n=6$ & $23.1 \pm 4.4$ & & $351.6 \pm 39.9$ & & $111.5 \pm 26.3$ & $220.1 \pm 35.8$ \\
\hline \multirow[t]{3}{*}{$D$} & $\mathrm{NR} n=10$ & $19.6 \pm 2.2$ & & $335.1 \pm 33.3$ & & & \\
\hline & $3 \mathrm{R} C \mathrm{Cnt} n=6$ & $29.9 \pm 7.9$ & & $371.3 \pm 35.1$ & & & \\
\hline & $3 R n=12$ & $17.1 \pm 1.9$ & & $650.7 \pm 65.1$ & & & \\
\hline \multirow[t]{3}{*}{$E$} & $\mathrm{NR} n=6$ & $26.7 \pm 2.6$ & $399.9 \pm 87.7$ & & & & \\
\hline & $3 R \operatorname{Ros} n=6$ & $28.3 \pm 4.0$ & $687.7 \pm 135.3$ & & & & \\
\hline & $3 \mathrm{R} T n=6$ & $25.0 \pm 3.5$ & $782.0 \pm 102.8$ & & & & \\
\hline \multicolumn{8}{|l|}{ Figure 2} \\
\hline \multirow[t]{4}{*}{ A } & $\mathrm{NR}+\operatorname{Veh} n=12$ & & $382.2 \pm 44.1$ & & & & \\
\hline & $N R+C y c n=12$ & & $357.5 \pm 56.1$ & & & & \\
\hline & $3 \mathrm{R}+$ Veh $n=13$ & & $596.1 \pm 57.7$ & & & & \\
\hline & $3 R+C y c n=10$ & & $100.8 \pm 18.6$ & & & & \\
\hline \multirow[t]{2}{*}{ B } & Veh $n=9$ & & & $506.7 \pm 54.6$ & $682.3 \pm 54.6$ & & \\
\hline & Cycn $=10$ & & & $218.8 \pm 70.5$ & $285.2 \pm 76.5$ & & \\
\hline \multirow[t]{2}{*}{$C$} & Veh $n=10$ & & $491.0 \pm 48.3$ & & & & \\
\hline & Cyc $n=9$ & & $555.9 \pm 45.4$ & & & & \\
\hline \multirow[t]{2}{*}{$D$} & Veh $n=7$ & & $494.9 \pm 100.2$ & & & & \\
\hline & $C y c n=7$ & & $529.3 \pm 101.4$ & & & & \\
\hline \multirow[t]{2}{*}{$E$} & $N R n=8$ & & $560.2 \pm 93.6$ & & & & \\
\hline & $3 \mathrm{R} n=8$ & & $540.7 \pm 86.4$ & & & & \\
\hline \multirow[t]{2}{*}{$F$} & Veh $n=12$ & & $494.6 \pm 52.0$ & & & & \\
\hline & Cycn $=13$ & & $503.1 \pm 46.4$ & & & & \\
\hline \multirow[t]{4}{*}{ Figure 3} & $\mathrm{NRT} 2 \mathrm{~d} n=11$ & & $303.5 \pm 43.1$ & & & & \\
\hline & $\mathrm{NR} T 20 \mathrm{~d} n=8$ & & $560.2 \pm 93.6$ & & & & \\
\hline & NR T55d $n=10$ & & $231.2 \pm 47.6$ & & & & \\
\hline & $3 \mathrm{R}$ T55d $n=10$ & & $546.2 \pm 76.1$ & & & & \\
\hline \multirow[t]{5}{*}{ Figure 4} & 1. $\mathrm{T} 1 n=8$ & & $459.9 \pm 106.9$ & & & & \\
\hline & 2. $\mathrm{T} 1 n=8$ & & $156.3 \pm 72.5$ & & & & \\
\hline & 2. $\mathrm{T} 2 n=8$ & & $430.5 \pm 103.9$ & & & & \\
\hline & 1. $\mathrm{T} 2 n=8$ & & $130.3 \pm 39.1$ & & & & \\
\hline & 1. $\mathrm{T} 3 n=8$ & & $541.8 \pm 115.4$ & & & & \\
\hline
\end{tabular}

Veh, Vehicle; (yc, cycloheximide; dash (-), not applicable.

mide compared with vehicle was found in the NR group, indicating that the effect of cycloheximide is contingent upon reactivation. Together, these data indicate that memory reactivation by nonreinforced retrievals makes the memory sensitive to protein synthesis inhibitors and leads to memory strengthening. Thus, reconsolidation accompanies memory strengthening.

\section{Reconsolidation is a phase of memory consolidation}

Does reactivation-induced memory strengthening also makes the memory more resistant to disruption, or, in other words, consolidated? We tested how sensitive a memory is to protein synthesis inhibitors after three reactivations. Rats were trained in IA and divided into two groups. One group remained in the home cage for $8 \mathrm{~d}$ (Fig. $2 \mathrm{~B}$ ), while the other underwent $3 \times 10$ s reactivations spaced by $2 \mathrm{~d}$ (Fig. $2 C$ ). Two days later, both groups were exposed to one $10 \mathrm{~s}$ reactivation and were treated with two subcutaneous injections of either cycloheximide or vehicle, one $30 \mathrm{~min}$ before reactivation and the second $5 \mathrm{~h}$ later. This protocol blocks $70 \%$ of protein synthesis for $>6 \mathrm{~h}$ and disrupts both memory consolidation and reconsolidation (Milekic et al., 2006; Taubenfeld et al., 2009). In these experiments, two cycloheximide injections were used to maximally disrupt protein synthesis. All rats were tested $2 \mathrm{~d}$ later. As shown in Figure 2, B and $C, 3 \times 10$ s reactivations made the memory resistant to disruption. Two-way ANOVA comparing the effect of treatment (cycloheximide) and reactiva- tions (shown in Fig. $2 B, C)$ revealed an effect of treatment $\left(F_{(1,34)}=\right.$ 4.304, $p=0.0457)$, reactivations $\left(F_{(1,34)}=8.937, p=0.0052\right)$, and a treatment $\times$ reactivations interaction $\left(F_{(1,34)}=10.77, p=\right.$ $0.0024)$. Bonferroni post hoc test showed that, compared with vehicle, cycloheximide disrupt a nonreactivated memory when given with testing $8 \mathrm{~d}$ after training $(p<0.001)$, but has no effect if during these $8 \mathrm{~d}$ the memory has been reactivated three times. Hence, reactivating the memory makes the memory resistant to disruption. As in the group that stayed in the home cage for $8 \mathrm{~d}$ without reactivation, cycloheximide disrupted memory retention $2 \mathrm{~d}$ later (T1); 24 h later, we administered a reminder shock and tested the retention a day later (T2) (Fig. 2B). A two-way ANOVA that compared the effect of treatment (cycloheximide/ vehicle) and test (T1 and T2) across groups revealed a significant treatment effect $\left(F_{(1,34)}=28.76, p<0.0001\right)$, no test effect $\left(F_{(1,34)}=\right.$ 3.589, $p=0.0667)$ and no interaction $\left(F_{(1,34)}=0.7312, p=\right.$ $0.3985)$. Bonferroni post hoc test showed that, compared with vehicle, cycloheximide significantly and persistently disrupted memory retention $(p<0.01)$. To test whether the memory loss in the cycloheximide-injected rats could be due to facilitated extinction, we determined whether memory could be reinstated by a reminder shock in a different context (Bouton, 1993). The following day, both vehicle- and cycloheximide-injected rats underwent this protocol and memory was re tested the next day. Memory retention was not reinstated $(p<0.001)$, indicating 
A
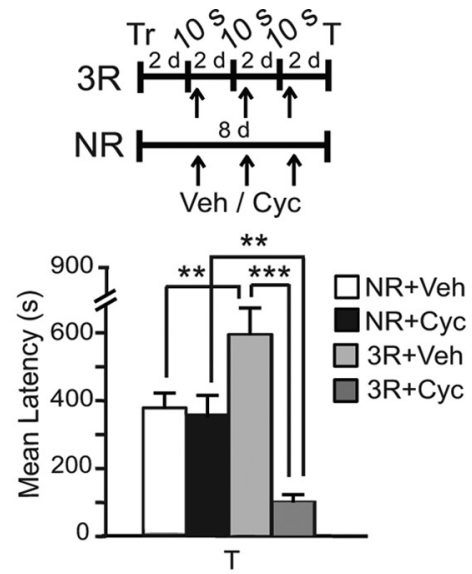

C
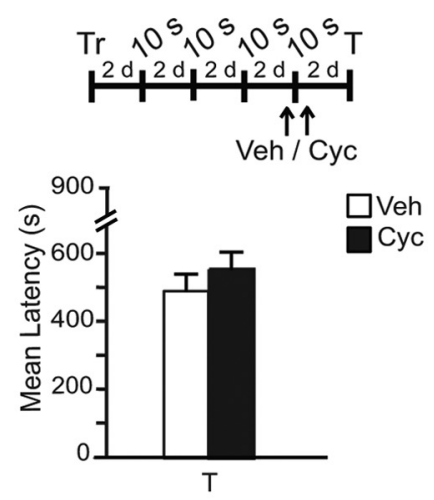

E
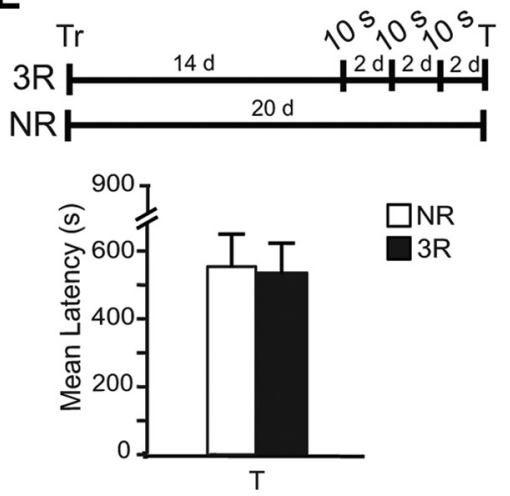

B
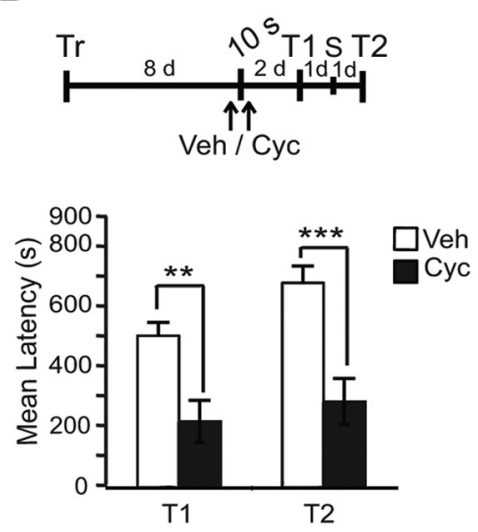

D
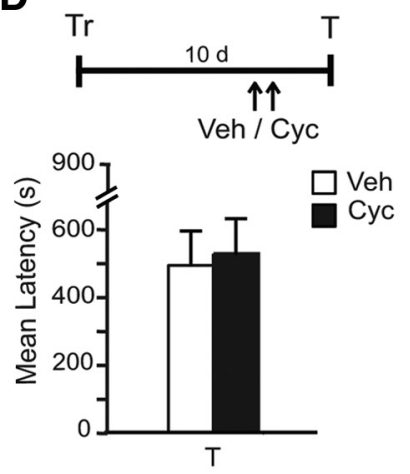

$\mathbf{F}$
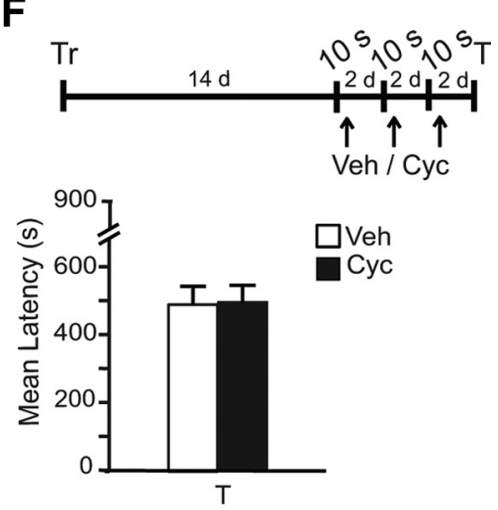

Figure 2. Recent but not remote memories are strengthen by reconsolidation and are susceptible to be disrupted by protein synthesis inhibitors (PSI). A, Experimental timelines are shown above each experiment. Memory acquisition (Tr) and retention are expressed as mean latency \pm SEM (in seconds, s). Rats were trained and underwent either $3 \times 10$ s reactivations starting $2 \mathrm{~d}$ after training or remained in the home cage (NR). Both groups were injected immediately after each reactivation, or at paired time points, with either cycloheximide (Cyc) or vehicle (Veh) and tested $8 \mathrm{~d}$ after training $(\boldsymbol{T})$. At T, $3 \times 10$ s reactivations significantly increased latency $\left.{ }^{* *} p<0.01\right)$. Cycloheximide significantly disrupted this latency ( ${ }^{* * *} p<0.001$ ), which was decreased below that of cycloheximide-NR $\left({ }^{* *} p<0.01\right) . \boldsymbol{B}$, Rats were trained and, $8 \mathrm{~d}$ after training, underwent one single 10 s reactivation and two injections (one $15 \mathrm{~min}$ before and one immediately after reactivation) of either cycloheximide or vehicle. At testing (T1), $2 \mathrm{~d}$ later, cycloheximide significantly disrupted latency compared with vehicle $\left({ }^{* *} p<0.01\right)$. Retention did not reinstated after a shock reminder $\left(S, T 2,{ }^{* * *} p<0.001\right)$. C, Rats followed the same protocol as in $(\boldsymbol{b})$, but received $3 \times 10$ s reactivations starting $2 \mathrm{~d}$ after training. Cycloheximide failed to affect latency at testing $(\boldsymbol{T})$. D, Compared with vehicle, cycloheximide, injected at matched time points as in $\boldsymbol{B}$ and $\boldsymbol{C}$, had no effect. $\boldsymbol{E}$, Rats that underwent either $3 \times 10$ s reactivations 2 weeks after training with an interreactivation interval of $2 \mathrm{~d}$ or NR had similar, strong latencies at testing, $20 \mathrm{~d}$ after training. $\boldsymbol{F}$, Rats underwent the same reactivation protocol as in $\boldsymbol{E}$ and were injected with either cycloheximide or vehicle after each reactivation. At testing $(\boldsymbol{T})$, no difference was found between groups.

that the latency loss is likely not due to extinction but rather reconsolidation disruption. The cycloheximide-dependent disruption seen in the home cage group was contingent upon reactivation, rats were trained and received double injection of either cycloheximide or vehicle in the absence of reactivation and were tested $2 \mathrm{~d}$ later. As shown in Figure $2 D$, no effect of treatment was found (Student's $t$ test $t_{(12)}=$ $0.2407, p=0.8139)$. In contrast, a Student's $t$ test that compared the retentions of cycloheximide- or vehicle-injected rats that underwent $3 \times 10 \mathrm{~s}$ reactivations (Fig. $2 C$ ) revealed no effect $\left(t_{(17)}=0.9715, p=\right.$ 0.3449).

Together, these data indicate that retrievals, which induce reconsolidation and memory strengthening, also make the memory resistant to disruption, therefore more consolidated.

\section{The reconsolidation-dependent} memory strengthening is temporally limited. The passage of time, like retrievals, leads to memory strengthening

Several laboratories, including ours, previously showed that the passage of time, as well as training intensity and reactivation duration, plays an important role in regulating reconsolidation (Milekic and Alberini, 2002; Eisenberg et al., 2003; Eisenberg and Dudai, 2004; Suzuki et al., 2004; Frankland et al., 2006; Bustos et al., 2009). Whereas 2and 7-d-old IA memories are sensitive to disruption following retrieval, 2- and 4-week-old memories are resistant, indicating that the passage of time renders memories increasingly resistant to post-retrieval disruption (Milekic and Alberini, 2002). Here we asked two questions: does time change the ability of the memory to undergo reconsolidation and, if this is the case, does this prevent reactivation-dependent memory strengthening?

To address these questions, rats were trained in IA, and 2 weeks after training, underwent either $3 \times 10$ s reactivations spaced by $2 \mathrm{~d}$, or remained in the home cage (NR). Memory was tested $20 \mathrm{~d}$ after training. As shown in Figure 2E, a Student's $t$ test that compared the latencies between groups showed no significant effect of reactivation $\left(t_{(14)}=0.1534, p=\right.$ 0.8803). Notably, the retention levels of both groups were higher compared with those of rats that remained in the home cage and were tested $8 \mathrm{~d}$ after training (Fig. 1D). Thus, with the passage of time, memory becomes stronger even in the absence of explicit retrievals. Furthermore, after the temporal-induced strengthening, memory is not further enhanced by multiple reactivations. Despite the fact that there was no reactivation-induced memory enhancement, we tested whether reactivations given 2 weeks after training render the memory sensitive to inhibition of protein synthesis. Rats underwent the same protocol and, immediately after each reac- 

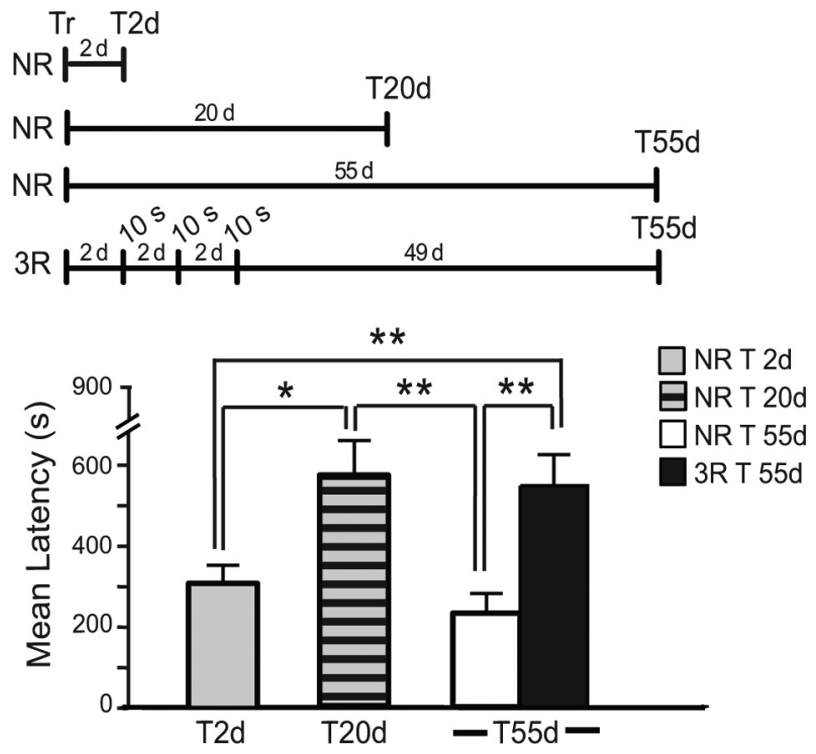

Figure 3. Multiple reactivations make the memory resistant to forgetting. Experimental timelines are shown above the experiment. Animals were trained and divided in 4 groups: one group was tested $2 \mathrm{~d}$ after training (NR T2d), the second was tested $20 \mathrm{~d}$ after training (NR T20d); the third was tested $55 \mathrm{~d}$ after training (NR T55d). The last group underwent $3 \times 10 \mathrm{~s}$ reactivations starting $2 \mathrm{~d}$ after training and with an interreactivation interval of $2 \mathrm{~d}$, and was tested $55 \mathrm{~d}$ after training (3R T55d). NR T20d had a significantly stronger latency compared with $N R T 2 d\left({ }^{*} p<0.05\right)$. The latency of NR T55d was significantly decreased compare with that of NR $\operatorname{T20d}\left({ }^{* *} p<0.01\right)$. This decay of latency was completely rescued in $3 R \operatorname{T5} 5 \mathrm{~d}\left({ }^{* *} p<0.01\right)$.

tivation, they received a systemic injection of either cycloheximide or vehicle, a treatment that disrupted the memory if delivered during the first week after training (Fig. $2 \mathrm{~A}$ ). Rats were tested $2 \mathrm{~d}$ after the last reactivation ( $20 \mathrm{~d}$ after training). A Student's $t$ test showed no effect of treatment $\left(t_{(23)}=0.1231, p=\right.$ 0.9031 , Fig. $2 F$ ). Thus, unlike the first week after training, multiple reactivations given to a 2 -week-old memory do not make the memory sensitive to disruption by protein synthesis inhibition.

We concluded that time, like multiple reactivations by retrieval during the first week, results in memory enhancement and strengthening. This memory is also insensitive to disruption by protein synthesis inhibition.

\section{Retrieval of a young memory prevents forgetting}

We then asked: do multiple reactivations given during the first week after training prevent forgetting? To address this question, rats underwent training and were divided into four groups. The first one remained in the home cage and was tested $2 \mathrm{~d}$ after training (NR T2d). The second remained in the home cage and was tested $20 \mathrm{~d}$ after training (NR T20d); the third group underwent $3 \times 10 \mathrm{~s}$ reactivations starting $2 \mathrm{~d}$ after training with an interreactivation interval of $2 \mathrm{~d}$ and was tested $55 \mathrm{~d}$ after training (3R T55d). The last group served as a NR control of the third group and remained in the home cage for $55 \mathrm{~d}$ before testing. As depicted in Figure 3, one-way ANOVA showed a significant group effect $\left(F_{(3,35)}=6.629, p=0.0012\right.$, $)$ and Neuman-Keuls post hoc test revealed that the latency of the 55-d-old memory had significantly decayed compared with that of a 20 -d-old memory $(p<0.01)$. This decay was completely prevented in the $3 \times 10 \mathrm{~s}$ reactivated group $(p<0.01)$. Hence, reactivating the memory with retrievals during the first week after training significantly prevents forgetting.
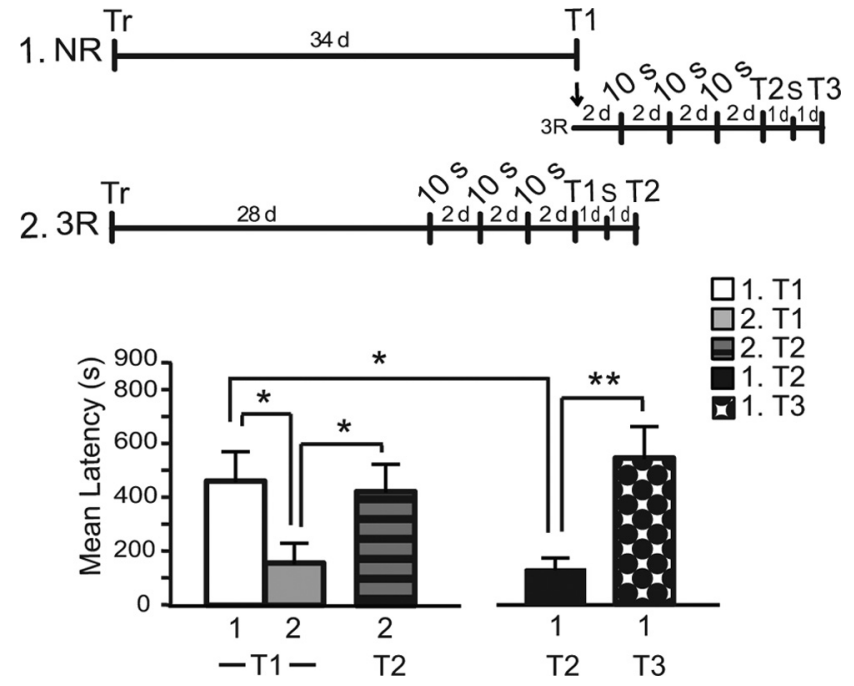

Figure 4. Retrievals of a 4-week-old memory lead to a facilitation of extinction. Experimental timelines are shown on top. The animals were trained and divided into two groups. The first group (1) was tested $34 \mathrm{~d}$ after training. The second group (2) underwent $3 \times 10$ s reactivations starting $28 \mathrm{~d}$ after training with an interreactivation interval of $2 \mathrm{~d}$. Both groups were tested $34 \mathrm{~d}$ after training (T1). At T1, group 2 had a significantly lower latency than group $1\left({ }^{*} p<0.05\right)$. group 2 underwent a footshock reminder (S) $1 \mathrm{~d}$ after T1 and was tested again $1 \mathrm{~d}$ later (T2). At $\mathrm{T} 2$, latency was significantly higher compared with $\mathrm{T} 1\left({ }^{*} p<0.05\right)$. The group 1 , after $\mathrm{T} 1$, underwent the same reactivation protocol as group 2 and was tested $2 \mathrm{~d}$ later (T2). This T2 latency was significantly lower than that of $\mathrm{T} 1\left({ }^{*} p<0.05\right)$. A reminder footshock (S), given $1 \mathrm{~d}$ later, resulted in a significant recovery of the latency at Test 3 the following day $\left({ }^{* *} p<0.01\right)$.

\section{Retrieval of an old memory leads to extinction}

As there is significant forgetting over weeks of an IA memory evoked by $0.6 \mathrm{~mA}$, we asked whether a weaker memory, whose retention has decayed over time, becomes again sensitive to disruption by protein synthesis inhibitors when reactivated and could be strengthened by multiple reactivations. Hence, we tested the effect of $3 \times 10$ s reactivation of a 28 -d-old memory. Rats were trained and divided into two groups. One received $3 \times 10$ s reactivations $28 \mathrm{~d}$ after training with an interreactivation interval of 2 $\mathrm{d}$, the other remained in the home cage (NR). All rats were tested $34 \mathrm{~d}$ after training (Fig. 4). At testing, the animals that received the reactivations had a significantly lower latency than those of the NR group (Student's $t$ test, $t_{(14)}=2.351, p=0.0339$ ), suggesting that reactivations induced extinction. To test this hypothesis, the rats of the $3 \times 10$ s reactivation group were exposed to a reminder shock in a different context. The reminder shock significantly reinstated memory retention (Student's $t$ test, $t_{(14)}=2.164, p=0.0483$ ), indicating that, indeed, reactivations led to extinction. To further confirm these results, the NR group underwent $3 \times 10$ s reactivations every $2 \mathrm{~d}$ after its testing and was tested again $2 \mathrm{~d}$ after the last reactivation ( $42 \mathrm{~d}$ after training). Similar to the previous group, the rats extinguished the memory following the reactivations (Student's $t$ test, $t_{(14)}=2.898, p=0.0117$ ) and reinstated it following a reminder shock (Student's $t$ test, $t_{(14)}=3.378, p=0.0045$ ). Thus, the same three reactivations that strengthen and consolidate a 1 -week-old memory have no effect on a 2 -week-old, consolidated memory and extinguish a 1-month-old memory.

Together, the present results show that, over time, the storage of long-term memory changes, as revealed by the consequences of its retrieval. While retrieval results in reconsolidation and strengthening of a young memory, it evokes extinction in an older one. 


\section{Discussion}

In this study, we have investigated the effect of memory retrievalinduced processes and their interaction with the passage of time. We find that retrievals of a young, 1-week-old IA memory results in reconsolidation that mediates memory strengthening and prevents forgetting. This effect is, however, temporally limited. Over time, even in the absence of explicit retrievals, memory strengthens and consolidates, and a 2-week-old IA memory becomes insensitive to both the strengthening effect of retrieval and postretrieval interference. Furthermore, at later times, retrievals of an older, 4-week-old IA memory, result in extinction. We conclude that a stored long-term memory is very dynamic, and its dynamic state dictates whether retrieval leads to either reconsolidation to consolidate memories or extinction. These findings have implications for designing clinical therapies based on either extinction or reconsolidation.

\section{Post-retrieval induced fragility correlates with memory strengthening}

Our results show that multiple brief memory reactivations with a protocol that preferentially evokes reconsolidation and not extinction lead to significant memory enhancement as a result of reconsolidation. This suggests that a function of reconsolidation is to mediate memory strengthening and prevent forgetting. This conclusion is in line with evidence reporting that memory can indeed be enhanced by pharmacological treatments given in combination with its reactivation (Frenkel et al., 2005; de Oliveira et al., 2007; Tronson and Taylor, 2007, Chen et al., 2010). In agreement with previous studies, our results show that CS exposures lead to either reconsolidation or extinction depending on the reactivation modalities, which likely occurs according to the hypothesis of trace dominance (Eisenberg et al., 2003). In fact, whereas multiple reactivations by testing separated by $2 \mathrm{~d}$ led to extinction, the same number of testing separated by 1 week resulted in memory strengthening.

In addition to the hypothesis tested here that reconsolidation mediates memory strengthening (Sara, 2000b), another nonmutually exclusive hypothesis has been proposed to explain the function of reconsolidation. It suggested that reconsolidation mediates memory updating, that is the integration of new information into the background of the past (Lewis, 1979; Sara, 2000a; Dudai, 2004). Studies focusing on testing this hypothesis led to contrasting conclusions, which, in part, may be due to a different use of the term memory updating. In previous studies, we concluded that memory updating, consisting of making new associations with a reactivated memory, does not recruit reconsolidation but a new, independent consolidation process (Tronel et al., 2005). Our conclusion was based on the use of a second order conditioning and selective interfering approaches that disrupted either reconsolidation or the consolidation of new traces. Others studies tested whether multiple learning trials, during a learning curve, evoke reconsolidation and use it to mediate additional learning, which they refereed to as memory updating. They found that posttrial applications of amnesic treatments disrupt memory retention only when the memory was not in an asymptotic level; however, when retention had reached an asymptotic level and no further learning or increased retention was evident, memory remained stable and resistant to disruption. Some authors showed that, if then new events are presented and associated with this stable memory, the old memory becomes labile and undergoes reconsolidation. The authors concluded that reconsolidation occurs to update the memory with the new information (Rodriguez-Ortiz et al., 2005, 2008; Morris et al., 2006; Winters et al., 2009). However, since both consolidation and reconsolidation are labile and sensitive to similar interferences, and these studies did not dissociate whether the updating is actually mediated by a new consolidation process that occurs simultaneously with the reconsolidation of the original trace, it still remains unclear whether reconsolidation mediates this type of memory updating.

To our knowledge, only one other study has tested whether the reconsolidation of a fear conditioning memory mediates memory strengthening by using as a reactivating event a second reinforced learning trial (Lee, 2008). The author reported that a second learning trial strengthens a contextual fear memory. Our present results, using nonreinforced contextual reactivation, reach the same conclusion. Moreover, we show that the IA reconsolidation-dependent memory strengthening is limited to young memories. This temporal window, which, with the training conditions used here (e.g., shock intensity of $0.6 \mathrm{~mA}$ ) lasts for $>1$ but $<2$ weeks after training, strictly overlaps with the temporal boundary of IA memory reconsolidation that we previously reported (Milekic and Alberini, 2002). Thus, the prerogative to undergo post-retrieval reconsolidation coincides with the temporal window during which memory can be strengthened. We will refer to this temporal window as the sensitive period. We conclude that, after the initial, post-encoding fragility, which lasts for $\sim 24 \mathrm{~h}$, memory lies in a sensitive period for some time (in the order of weeks). During this sensitive period, the strength of the memory can be bidirectionally changed if memory is reactivated for example by retrieval: memory reconsolidates to strengthen, or it can be weakened if the process of reconsolidation is disrupted.

\section{Reconsolidation and time contribute to memory consolidation}

We found that the passage of time leads to memory strengthening and resilience to disruption even in the absence of explicit reactivations. This increased strength in the response that occurs with time, also known as incubation effect, has been reported by several previous studies to accompany fear memories and perceptual learning, as well as craving of drugs of abuse after withdrawal (McAllister and McAllister, 1967; Eysenck, 1968; Karni et al., 1994; Grimm et al., 2001; Pickens et al., 2009). Our results show that not only a 2 -week-old IA memory is stronger than a 2-d-old memory, but that it has become resistant to post-retrieval protein synthesis inhibition and its retrieval does not further strengthen retention. Furthermore, we show that reactivations accelerate this process and, indeed, a 1-week-old memory that underwent three reactivations becomes resistant to post-retrieval interference. It is tempting here to speculate that the passage of time may account for implicit reactivations perhaps during sleep (Stickgold and Walker, 2007) or regulated by circadian rhythms (EckelMahan and Storm, 2009); however, the underlying mechanisms still remain to be established. Similarly, an intriguing hypothesis that remains to be investigated is whether the sensitive period reflects the reorganization of the memory trace; that is, memory becomes less dependent from the hippocampus while increasingly more dependent on cortical representation (Squire and Bayley, 2007) or increased distribution over brain areas (Nadel and Moscovitch, 1997).

Our results support the idea proposed a few years ago by both Dudai and Eisenberg (2004) and Alberini (2005) that reconsolidation is an integral part of the consolidation process. In other words, reconsolidation is one contributing phase of a lingering consolidation process. 
Interestingly, although they offered a different interpretation, the studies that investigated reactivations by multiple training trials in a learning curve (Rodriguez-Ortiz et al., 2005; Morris et al., 2006) reached similar outcomes; that is, when memory in a nonasymptotic level becomes labile after reactivation and strengthens through reconsolidation. We suggest that their view, that the stabilization and strengthening of a multiple trials task is mediated by reconsolidations, is highly reminiscent of the process that accompanies a single IA trial learning that, over time, develops a graded increased resistance to reconsolidation disruption. Notably, this process of increased resistance to disruption with the passage of time coincides with the definition of consolidation provided by the classical hypothesis (McGaugh, 2000; Squire, 2009); in other words, a process that strengthens memory and makes them resistant to disruption. However, the classical hypothesis of consolidation did not account for, nor explored, the mechanistic contributions of each trace reactivation elicited by single learning trials, or the fact that nonreinforced retrievals can produce a similar result.

\section{The same retrievals that reconsolidate a young memory extinguish an older memory}

The same behavioral retrievals that strengthen a 1-week-old memory and has no effect on a 2-week-old, strong memory extinguish an older, 4-week-old memory. Hence, the stored memory is very dynamic and undergoes significant changes over time, even without explicit retrievals. It is important that further studies elucidate the mechanisms that over time lead to favor extinction. Extinction is a new learning, and, as such, it undergoes a consolidation process that is sensitive to the same amnestic interferences as the consolidation or reconsolidation of the original memory (Quirk and Mueller, 2008). There is a great deal of interest in identifying therapeutic approaches based on either reconsolidation or extinction to weaken traumatic memories or other trauma-induced pathologies, such as posttraumatic stress disorder (Davis et al., 2006; Kindt et al., 2009; Schiller et al., 2010). As blocking reconsolidation or extinction would result in opposite outcomes, understanding which process is evoked by retrieval is key for designing efficacious treatments. Our data also show that it is not the retention level of the memory that dictates whether CS presentation leads to either reconsolidation or extinction: in fact, the retention level of both, the 2-week-old memory undergoing reconsolidation and strengthening and the 4-week-old memory undergoing extinction were comparable.

Although further studies are needed to understand the mechanisms that accompany the evolution of memory storage, we speculate that possible contributing mechanisms are implicit trace reactivations that can occur during both the sleep or awake cycles (Karni et al., 1994; Stickgold and Walker, 2007; Karlsson and Frank, 2009).

We propose that the storage of information is very dynamic and that its temporal evolution regulates behavioral outcomes. This knowledge is critical for potential clinical applications.

\section{References}

Alberini CM (2005) Mechanisms of memory stabilization: are consolidation and reconsolidation similar or distinct processes? Trends Neurosci 28:51-56.

Alberini CM (2008) The role of protein synthesis during the labile phases of memory: revisiting the skepticism. Neurobiol Learn Mem 89:234-246.

Alberini CM (2009) Transcription factors in long-term memory and synaptic plasticity. Physiol Rev 89:121-145.

Boccia M, Freudenthal R, Blake M, de la Fuente V, Acosta G, Baratti C, Romano
A (2007) Activation of hippocampal nuclear factor-kappa B by retrieval is required for memory reconsolidation. J Neurosci 27:13436-13445.

Bouton ME (1993) Context, time, and memory retrieval in the interference paradigms of Pavlovian learning. Psychol Bull 114:80-99.

Bustos SG, Maldonado H, Molina VA (2009) Disruptive effect of midazolam on fear memory reconsolidation: decisive influence of reactivation time span and memory age. Neuropsychopharmacology 34:446-457.

Chen DY, Stern SA, Garcia-Osta A, Saunier-Rebori B, Pollonini G, BambahMukku D, Blitzer RD, Alberini CM (2010) A critical role for IGF-II in memory consolidation and enhancement. Nature, in press.

Davis HP, Squire LR (1984) Protein synthesis and memory: a review. Psychol Bull 96:518-559.

Davis M, Barad M, Otto M, Southwick S (2006) Combining pharmacotherapy with cognitive behavioral therapy: traditional and new approaches. J Trauma Stress 19:571-581.

Dawson RG, McGaugh JL (1969) Electroconvulsive shock effects on a reactivated memory trace: further examination. Science 166:525-527.

Debiec J, LeDoux JE, Nader K (2002) Cellular and systems reconsolidation in the hippocampus. Neuron 36:527-538.

de Oliveira LF, Camboim C, Diehl F, Consiglio AR, Quillfeldt JA (2007) Glucocorticoid-mediated effects of systemic oxytocin upon memory retrieval. Neurobiol Learn Mem 87:67-71.

Dudai Y (2004) The neurobiology of consolidations, or, how stable is the engram? Annu Rev Psychol 55:51-86.

Dudai Y (2006) Reconsolidation: the advantage of being refocused. Curr Opin Neurobiol 16:174-178.

Dudai Y, Eisenberg M (2004) Rites of passage of the engram: reconsolidation and the lingering consolidation hypothesis. Neuron 44:93-100.

Eckel-Mahan KL, Storm DR (2009) Circadian rhythms and memory: not so simple as cogs and gears. EMBO Rep 10:584-591.

Eisenberg M, Dudai Y (2004) Reconsolidation of fresh, remote, and extinguished fear memory in Medaka: old fears don't die. Eur J Neurosci 20:3397-3403.

Eisenberg M, Kobilo T, Berman DE, Dudai Y (2003) Stability of retrieved memory: inverse correlation with trace dominance. Science 301:1102-1104.

Eysenck HJ (1968) A theory of the incubation of anxiety/fear responses. Behav Res Ther 6:309-321.

Frankland PW, Bontempi B (2005) The organization of recent and remote memories. Nat Rev Neurosci 6:119-130.

Frankland PW, Ding HK, Takahashi E, Suzuki A, Kida S, Silva AJ (2006) Stability of recent and remote contextual fear memory. Learn Mem 13:451-457.

Frenkel L, Maldonado H, Delorenzi A (2005) Memory strengthening by a real-life episode during reconsolidation: an outcome of water deprivation via brain angiotensin II. Eur J Neurosci 22:1757-1766.

Grimm JW, Hope BT, Wise RA, Shaham Y (2001) Neuroadaptation. Incubation of cocaine craving after withdrawal. Nature 412:141-142.

Hupbach A, Gomez R, Hardt O, Nadel L (2007) Reconsolidation of episodic memories: a subtle reminder triggers integration of new information. Learn Mem 14:47-53.

Karlsson MP, Frank LM (2009) Awake replay of remote experiences in the hippocampus. Nat Neurosci 12:913-918.

Karni A, Tanne D, Rubenstein BS, Askenasy JJ, Sagi D (1994) Dependence on REM sleep of overnight improvement of a perceptual skill. Science 265:679-682.

Kindt M, Soeter M, Vervliet B (2009) Beyond extinction: erasing human fear responses and preventing the return of fear. Nat Neurosci 12:256-258.

Lee JL (2008) Memory reconsolidation mediates the strengthening of memories by additional learning. Nat Neurosci 11:1264-1266.

Lee JL (2009) Reconsolidation: maintaining memory relevance. Trends Neurosci 32:413-420.

Lewis DJ (1979) Psychobiology of active and inactive memory. Psychol Bull 86:1054-1083.

McAllister DE, McAllister WR (1967) Incubation of fear: an examination of the concept. J Exp Res Person 2:180-190.

McGaugh JL (2000) Memory-a century of consolidation. Science 287:248-251.

Medina JH, Bekinschtein P, Cammarota M, Izquierdo I (2008) Do memories consolidate to persist or do they persist to consolidate? Behav Brain Res 192:61-69. 
Milekic MH, Alberini CM (2002) Temporally graded requirement for protein synthesis following memory reactivation. Neuron 36:521-525.

Milekic MH, Brown SD, Castellini C, Alberini CM (2006) Persistent disruption of an established morphine conditioned place preference. J Neurosci 26:3010-3020.

Morris RG, Inglis J, Ainge JA, Olverman HJ, Tulloch J, Dudai Y, Kelly PA (2006) Memory reconsolidation: sensitivity of spatial memory to inhibition of protein synthesis in dorsal hippocampus during encoding and retrieval. Neuron 50:479-489.

Moscovitch M, Nadel L, Winocur G, Gilboa A, Rosenbaum RS (2006) The cognitive neuroscience of remote episodic, semantic and spatial memory. Curr Opin Neurobiol 16:179-190.

Nadel L, Moscovitch M (1997) Memory consolidation, retrograde amnesia and the hippocampal complex. Curr Opin Neurobiol 7:217-227.

Nader K, Schafe GE, LeDoux JE (2000) The labile nature of consolidation theory. Nat Rev Neurosci 1:216-219.

Pedreira ME, Maldonado H (2003) Protein synthesis subserves reconsolidation or extinction depending on reminder duration. Neuron 38:863-869.

Pickens CL, Golden SA, Adams-Deutsch T, Nair SG, Shaham Y (2009) Long-lasting incubation of conditioned fear in rats. Biol Psychiatry 65:881-886.

Power AE, Berlau DJ, McGaugh JL, Steward O (2006) Anisomycin infused into the hippocampus fails to block "reconsolidation" but impairs extinction: the role of re-exposure duration. Learn Mem 13:27-34.

Quirk GJ, Mueller D (2008) Neural mechanisms of extinction learning and retrieval. Neuropsychopharmacology 33:56-72.

Rodriguez-Ortiz CJ, De la Cruz V, Gutiérrez R, Bermudez-Rattoni F (2005) Protein synthesis underlies post-retrieval memory consolidation to a restricted degree only when updated information is obtained. Learn Mem 12:533-537.

Rodriguez-Ortiz CJ, Garcia-DeLaTorre P, Benavidez E, Ballesteros MA, Bermudez-Rattoni F (2008) Intrahippocampal anisomycin infusions disrupt previously consolidated spatial memory only when memory is updated. Neurobiol Learn Mem 89:352-359.
Sara SJ (2000a) Retrieval and reconsolidation: toward a neurobiology of remembering. Learn Mem 7:73-84.

Sara SJ (2000b) Strengthening the shaky trace through retrieval. Nat Rev Neurosci 1:212-213.

Schiller D, Monfils MH, Raio CM, Johnson DC, Ledoux JE, Phelps EA (2010) Preventing the return of fear in humans using reconsolidation update mechanisms. Nature 463:49-53.

Squire LR (2009) Memory and brain systems: 1969-2009. J Neurosci 29:12711-12716.

Squire LR, Bayley PJ (2007) The neuroscience of remote memory. Curr Opin Neurobiol 17:185-196.

Stickgold R, Walker MP (2007) Sleep-dependent memory consolidation and reconsolidation. Sleep Med 8:331-343.

Suárez LD, Smal L, Delorenzi A (2010) Updating contextual information during consolidation as result of a new memory trace. Neurobiol Learn Mem 93:561-571.

Suzuki A, Josselyn SA, Frankland PW, Masushige S, Silva AJ, Kida S (2004) Memory reconsolidation and extinction have distinct temporal and biochemical signatures. J Neurosci 24:4787-4795.

Taubenfeld SM, Milekic MH, Monti B, Alberini CM (2001) The consolidation of new but not reactivated memory requires hippocampal $\mathrm{C} / \mathrm{EBP}$ beta. Nat Neurosci 4:813-818.

Taubenfeld SM, Riceberg JS, New AS, Alberini CM (2009) Preclinical assessment for selectively disrupting a traumatic memory via postretrieval inhibition of glucocorticoid receptors. Biol Psychiatry 65:249-257.

Tronel S, Milekic MH, Alberini CM (2005) Linking new information to a reactivated memory requires consolidation and not reconsolidation mechanisms. PLoS Biol 3:e293.

Tronson NC, Taylor JR (2007) Molecular mechanisms of memory reconsolidation. Nat Rev Neurosci 8:262-275.

Winters BD, Tucci MC, DaCosta-Furtado M (2009) Older and stronger object memories are selectively destabilized by reactivation in the presence of new information. Learn Mem 16:545-553. 